\title{
Univ.-Prof. Dr. med. Christiane Kuhl erhält Gold-Medaille der International Society of Magnetic Resonance in Medicine (ISMRM)
}

Aachen/Toronto, 29.06.2015 - Univ.Prof. Dr. med.Christiane Kuhl, Direktorin der Klinik für Diagnostische und Interventionelle Radiologie der Uniklinik RWTH Aachen, ist von der International Society of Magnetic Resonance in Medicine (ISMRM) mit der Gold Medal ausgezeichnet worden. Die Fachgesellschaft für die Weiterentwicklung und die klinische und wissenschaftliche Anwendung der Magnet-Resonanz-Verfahren (u.a. Kernspintomografie) verleiht ihre höchste Auszeichnung an herausragende Persönlichkeiten auf dem Gebiet der MR-Forschung. Die feierliche Preisübergabe erfolgte Anfang Juni im Rahmen der ISMRM-Jahrestagung in Toronto, Kanada.

Die Preisträgerin, Univ.-Prof. Dr. med. Christiane Kuhl, steht mit dieser Auszeichnung in einer Riege mit den Nobelpreisträgern Richard R. Ernst, Sir Peter Mansfield und Paul Lauterbur. Mit Prof. Kuhl wurde nach Prof. Hedvig Hrzicak (Memorial Sloan Kettering Cancer Center, New York, USA) erst der 2. weiblichen Wissenschaftlerin überhaupt diese hohe Ehre zuteil. Der Preis wurde bislang selten

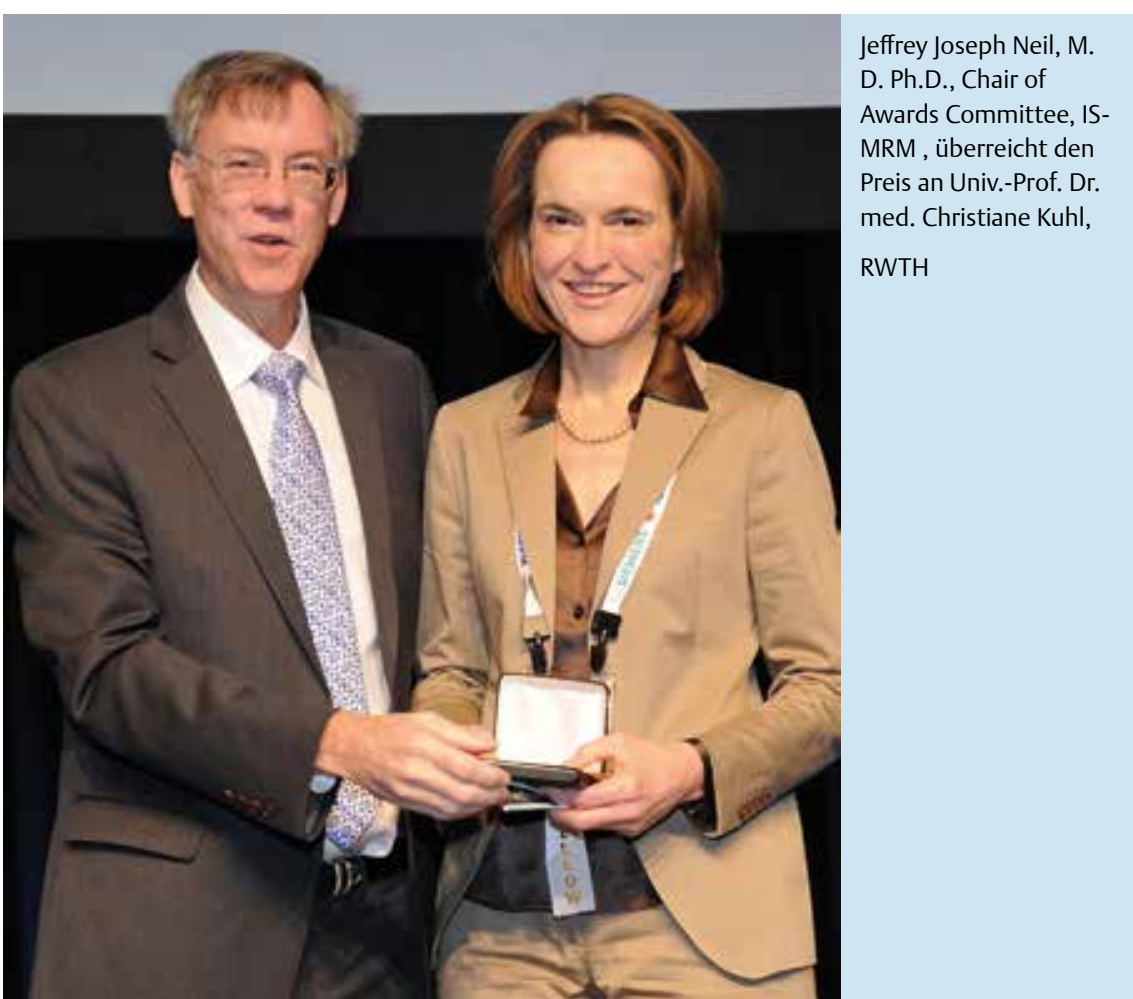

Jahren mit der MR-Diagnostik von Tumorerkrankungen, hier insbesondere von Brustkrebs. Erst vor Kurzem stellten Prof. die Durchführung einer Mammografie entspricht, aber eine bis zu 3-fach höhere Sicherheit im Nachweis von Brustkrebs bietet und ohne Röntgenstrahlen auskommt. Mittels dieses neuen Verfahrens konnte Brustkrebs gefunden werden, der weder in der Mammografie noch im Ultraschall vermutet oder erkannt worden war. Damit gilt das Verfahren als Meilenstein für die Brustkrebs-Früherkennung. Studien, die diesen Ansatz aufgreifen, lau- fen derzeit in den USA, England, Kanada und den Niederlanden an.

\section{Über die ISMRM \\ $\nabla$}

Die disziplinübergreifende Fachgesellschaft setzt sich aus Grundlagenwissenschaftlern, Ingenieuren, Physikern, Biologen und Medizinern zusammen. Aus den medizinischen Fachrichtungen sind im wesentlichen Radiologen, Kardiologen und Neurowissenschaftler vertreten. Die ISMRM hat über 8000 Mitglieder in 59 Ländern weltweit. Ihre Jahrestagung ist für alle im Bereich der Magnet-ResonanzVerfahren wissenschaftlich Tätigen eine Pflichtveranstaltung. Kuhl und ihr Team einen neuen Ansatz zur Früherkennung von Brustkrebs mittels MRT vor, der mit einer sehr kurzen Untersuchungszeit von nur 3 Minuten auskommt - was dem zeitlichen Aufwand für

\section{Über die Uniklinik RWTH Aachen (AöR) \\ $\nabla$}

Die Uniklinik RWTH Aachen verbindet als Supramaximalversorger patientenorientierte Medizin und Pflege, Lehre sowie Forschung auf internationalem Niveau. Mit 34 Fachkliniken, 25 Instituten und 5 fachübergreifenden Einheiten deckt die Uniklinik das gesamte medizinische Spektrum ab. Hervorragend qualifizierte Teams aus Ärzten, Pflegern und Wissenschaftlern setzen sich kompetent für die Gesundheit der Patienten ein. Die Bündelung von Krankenversorgung, Forschung und Lehre in einem Zentralgebäude bietet beste Voraussetzungen für einen intensiven interdisziplinären Austausch und eine enge klinische und wissenschaftliche Vernetzung. Rund 6000 Mitarbeiterinnen und Mitarbeiter sorgen für patientenorientierte Medizin und eine Pflege nach anerkannten Qualitätsstandards.

Die Uniklinik versorgt mit 1400 Betten rund 45000 stationäre und 200000 ambulante Fälle im Jahr.

\section{Pressekontakt}

Universitätsklinikum Aachen (AöR)

Dr. Mathias Brandstädter

Leitung Unternehmenskommunikation Pauwelsstraße 30

52074 Aachen

Tel.: $024180-89893$

Fax: 0241 80-3389893

mbrandstaedter@ukaachen.de 